\title{
"Des souvenirs dormant dans cette chevelure"... Étude de la chevelure de sainte Madeleine dans la littérature contemporaine
}

Katherine Rondou

\section{(2) OpenEdition}

Journals

\section{Édition électronique}

URL : http://journals.openedition.org/studifrancesi/6503

DOI : 10.4000/studifrancesi.6503

ISSN : 2421-5856

Éditeur

Rosenberg \& Sellier

\section{Édition imprimée}

Date de publication : 1 septembre 2010

Pagination : 232-243

ISSN : 0039-2944

\section{Référence électronique}

Katherine Rondou, «"Des souvenirs dormant dans cette chevelure"... Étude de la chevelure de sainte Madeleine dans la littérature contemporaine », Studi Francesi [En ligne], 161 (LIV | II) | 2010, mis en ligne le 30 novembre 2015, consulté le 08 janvier 2021. URL : http://journals.openedition.org/ studifrancesi/6503; DOI : https://doi.org/10.4000/studifrancesi.6503

\section{(c) (i) (9)}

Studi Francesi è distribuita con Licenza Creative Commons Attribuzione - Non commerciale - Non opere derivate 4.0 Internazionale. 


\section{"Des souvenirs dormant dans cette chevelure"... Étude de la chevelure de sainte Madeleine dans la littérature contemporaine}

Témoin d'une époque où le culte érotique du cheveu culmine ${ }^{1}$, le poème de Baudelaire rappelle combien la chevelure féminine a profondément marqué l'imaginaire. Son puissant caractère sexuel génère, en effet, de multiples fantasmes, dans la plupart des cultures ${ }^{2}$. Nous retrouvons donc, sans surprise, les longues mèches associées à la plus célèbre sainte pécheresse du christianisme, Marie-Madeleine, et ce, dès ses premières représentations, tant littéraires qu'iconographiques.

L'hagiographie assigne à chaque saint des attributs spécifiques. Ils assument une fonction à la fois de célébration, d'ancrage symbolique et d'identification. La chevelure constituera, avec les larmes et le parfum, l'un des principaux emblèmes magdaléens, dont la signification et l'importance évolueront au fil des siècles.

Figure synthétique ${ }^{3}$, la Madeleine occidentale naît au $\mathrm{VI}^{\mathrm{e}}$ siècle de la fusion, opérée par Grégoire le Grand, de trois figures évangéliques. (1) L'anonyme du repas chez Simon dans les synoptiques: ${ }^{4}$ notons que seul Luc la qualifie de pécheresse. (2) Marie de Béthanie: modèle de contemplation ${ }^{5}$, elle accomplit l'onction dans le quatrième évangile 6 . Enfin, (3) Marie de Magdala, la disciple fidèle. Libérée de sept démons par le Christ', elle l'accompagne au Golgotha ${ }^{8}$ et sera, au matin de Pâques, le premier témoin de sa Résurrection?. Dans ces différents épisodes, la chevelure n'apparait cependant qu'à deux reprises, lors des onctions lucanienne et johannique, où la jeune femme essuie les pieds du Messie de ses cheveux. Les longues mèches de la sainte connaitront pourtant une importante postérité, certainement en raison des connotations sexuelles que nous avons évoquées. Très vite, la théologie et les arts s'emparent de la scène, et se focalisent essentiellement sur le pardon accordé à la pécheresse, chez Luc. Étendard de sa sexualité dévoyée dès l'époque médiévale, la chevelure de Madeleine s'avilit aux pieds de Jésus, afin de manifester son repentir. Les mèches tentatrices cachent désormais le corps obscène, offrent une bure à la pénitente ${ }^{10}$. Rappelons que

(1) MARINA WARNER, Le vil et le vigoureux, la toison et le poil: des cheveux et de leur langage, traduit de l'anglais par Marie-Ange DutARTRE, dans MARIELaure Bernadac et Bernard Marcade, Fémininmasculin. Le sexe de l'art, Paris, Gallimard-Electa, 1996, p. 309.

(2) Jean Chevalier et Alain Gheerbrant, «Cheveux», dans Dictionnaire des symboles, Paris, Editions Robert Laffont S.A. et Editions de Jupiter, 1982 (1969), p. 234-p. 237; Michel CaZENAVE, «Cheveu», dans Encyclopédie des symboles, Paris, Le Livre de Poche, 1996, p. 132-p. 133.

(3) Pour une analyse complète de la question des trois Maries dans la littérature contemporaine, voir Katherine Rondou, Échos de la Madeleine, figure évangélique, dans la littérature contemporaine, «Rivista di Storia e Letteratura Religiosa», 2005, V. 41, fasc. 3, p. 413-p. 432.
(4) Mt 26, 6-13; Mc 14, 3-9; Lc 7, 36-50.

(5) Lc 10, 38-42.

(6) In 12, 1-11.

(7) Lc 8, 2; Mc 16, 9.

(8) Mt 28, 55-56; Mc 15, 40 et Jn 19, 25. Luc ne la mentionne pas et évoque uniquement «des femmes qui l'avaient suivi depuis la Galilée» (Lc 23, 49).

(9) Mt 28, 1-8; Mc 16, 1-8; Lc 24, 1-11 et Jn 20, $1-18$

(10) Estrella Ruiz-Galvez, Une chevelure my thique. Les cheveux de Madeleine, enseigne du féminin et emblème d'un repentir. Illustrations littéraires et représentations iconographiques d'un thème (XV'-XVII siècles), dans: AlaIn MonTandon, Marie-Madeleine, figure mythique dans la littérature et les arts, Clermont-Ferrand, Presses universitaires Blaise Pascal, 1999, p. 75-p. 86. 
si aucun évangéliste ne décrit cette chevelure, la tradition insistera continuellement sur son ampleur, sans doute par contamination avec une autre pécheresse repentie, sainte Marie l'Egyptienne. Sa légende a largement influencé l'hagiographie magdalénienne: la vie anachorétique de Marie-Madeleine à la Sainte-Baume doit beaucoup à la retraite au désert de la courtisane d'Alexandrie. Or, la Vita de l'Egyptienne précise que l'ermite se présenta au prêtre Zosime vêtue de ses seuls cheveux ${ }^{11} \ldots$

La chevelure lascive de Marie-Madeleine prolonge évidemment son pouvoir séducteur au-delà du Moyen-Âge. «D’une manière générale, le cheveu acquiert au lendemain de la Contre-Réforme un vague relent de sensualité et de péché» ${ }^{12}$. Nous avons cependant noté, chez quelques auteurs contemporains, une rupture avec l'image dichotomique imposée à l'attribut par la théologie. Celle-ci distingue d'une part la riche coiffure de la pécheresse, chargée sexuellement; d'autre part, la chevelure-cilice de la pénitente, mode d'expression de son repentir. Cette évolution, loin d'être généralisée, mérite néanmoins de retenir notre attention.

Notre corpus, constitué d'environ cent cinquante oeuvres, se veut le plus large possible et, sans prétendre à l'exhaustivité, constitue un ensemble représentatif de la littérature magdaléenne des $\mathrm{XX}^{\mathrm{e}}$ et $\mathrm{XXI}^{\mathrm{e}}$ siècles. Ceci suppose évidemment la prise en considération d'auteurs de langues, de formations, d'impacts, de cultures, et de champs littéraires très différents. Des «monuments» de la littérature internationale côtoieront donc des romanciers appréciés du grand public, ou encore des auteurs dont l'histoire littéraire peine à retenir le nom.

Tous nos auteurs n'exploitent pas le célèbre attribut, souvent en raison de la brièveté de l'œuvre ou de l'apparition de Madeleine (alors, simple personnage secondaire). Néanmoins, la chevelure demeure généralement très présente dans la littérature contemporaine, notamment dans le célèbre épisode de l'onction.

Parallèlement à la question des trois Maries, les Pères de l'Eglise se sont interrogés sur le nombre d'onction(s) ${ }^{13}$. Interpellés par des divergences apparaissant dans le troisième évangile, certains distinguent deux épisodes. L'onction de Galilée, relatée par Luc, traduit la contrition et la tendresse de la pécheresse qui, contrairement au Pharisien, accomplit les gestes d'hospitalité. Celle de Béthanie, évoquée par les autres évangélistes, s'apparente à une extrême-onction et souligne, avec les autres annonces de la Passion, l'approche du sacrifice christique. Il va sans dire que la chevelure véhicule des connotations bien différentes dans l'un et l'autre cas, et que la tradition de la pécheresse repentie s'est essentiellement inspirée de Lc 7, 36-50.

Une tradition dont nous trouvons encore largement trace au $\mathrm{Xx}^{\mathrm{e}}$ siècle, notamment dans une nouvelle de 1936 de Marguerite Yourcenar, Madeleine ou le Salut. Jean, plus soucieux de l'enseignement du Christ que de l'amour de sa fiancée, abandonne Madeleine au soir de ses noces ${ }^{14}$. Afin de le discréditer aux yeux du futur évangéliste,

(11) Pour une étude des rapports liant les légendes des deux saintes, voir: ELISABETH PinTo-MATHIEU, Trois vies de pécheresses repenties. Les saintes Marie l'Egyptienne, Marie-Madeleine et Thaïs, «Revue des Sciences humaines», juillet-septembre 1998, V. 251, p. 89-p. 109.

(12) Marina Warner, op. cit., p. 311.

(13) Pour une vue d'ensemble sur l'évolution de la querelle jusqu'au XIX ${ }^{e}$ siècle, le lecteur pourra se référer, à condition de faire preuve d'un minimum d'esprit critique, à l'ouvrage de l'abbé FaILLON (Monuments inédits sur l'apostolat de sainte MarieMadeleine en Provence, et sur les autres apôtres de cette contrée, saint Lazare, saint Maximin, sainte Marthe et les saintes Maries Jacobé et Salomé, Paris,
Ateliers catholiques du Petit-Rouge, 1848). L'étude de Jean PIROT (Trois amies de Jésus de Nazareth, Paris, Cerf, 1986) constitue une bonne synthèse pour le $\mathrm{Xx}^{\mathrm{e}}$ siècle.

(14) Selon Susan Haskins (Mary Magdalen, Myth and Metaphor, New York, Riverhead Books, 1993), l'abandon de Madeleine à Cana, au soir de ses noces, apparaît pour la première fois au $\mathrm{V}^{\mathrm{e}}$ siècle, chez saint Augustin. Un récit dont fait également mention la Légende dorée, mais pour le réfuter: «Certains auteurs racontent que Marie-Madeleine était la fiancée de saint Jean l'Evangéliste, et que celui-ci s'apprêtait à l'épouser lorsque le Christ, survenant au milieu de ses noces, l'appela à lui: ce dont $\mathrm{Ma}$ deleine fut si indignée que, depuis lors, elle se livra 
la jeune femme décide de séduire Jésus... avant de succomber, à son tour, à la parole divine. Le déploiement de ses charmes devient glorification du Messie: ses cheveux recouvrent «la nudité de [sa] faute» ${ }^{15}$. Dans son roman largement controversé, La dernière tentation du Christ (1955), l'écrivain et philosophe grec Nikos Kazantzaki souligne également la pudeur retrouvée de la convertie, par le biais de la chevelure. Lorsque Jésus, après un premier voyage en Galilée, revient chercher sa cousine à Magdala, celle-ci se jette à ses pieds et sa chevelure «encore imprégnée des anciens parfums, des parfums maudits» se répand sur son dos. Le Christ l'aide à se relever et Madeleine cache sa poitrine de ses longues mèches, avant d'accomplir l'onction ${ }^{16}$.

Parallèlement à ces chevelures éclipsant une sensualité désormais désavouée, plusieurs auteurs marquent la conversion de leurs héroïnes par le port d'un voile. En 1902, paraît Le rayon, scènes évangéliques de Marie Reynès-Monlaur ${ }^{17}$, une écrivain catholique française mineure, essentiellement active durant la première moitié du Xx siècle. Une fois l'onction accomplie, son héroïne hésite à sortir en cheveux: la narratrice lui offre son voile ${ }^{18}$. La chevelure ne dissimule plus un corps impudique, elle devient l'impudeur à masquer.

Lc 7, 36-50 évoque bien sûr le repentir, mais aussi l'affection de «celle qui a montré beaucoup d'amour». Déjà présente chez les auteurs cités, cette double composante apparaît nettement dans Jésus et Marie-Madeleine, correspondance intime (2001), un roman épistolaire du Français Bernard-G. Landry. Outre l'onction où Jésus pardonne à la jeune femme ${ }^{19}$, Landry se plaît à souligner l'attachement de la disciple par son habitude de caresser, de ses cheveux, les pieds du Messie ${ }^{20}$.

Cependant, quelques romanciers contemporains ne retiennent que le geste de douceur, et valorisent ainsi la «sainte amante» au détriment de la pécheresse repentie. Une originalité dont témoigne, entre autres, Jacqueline Kelen. Licenciée en lettres classiques et productrice à France Culture, la Française a consacré une part non négligeable de ses publications à Marie de Magdala, essentiellement des méditations personnelles ${ }^{21}$. Elle ne met en scène l'amie du Christ qu'à une seule reprise, dans Marie-Madeleine, un amour infini (1982). Dans ce roman, l'onction de Galilée célèbre le bien-aimé.

Avec le sel de mes yeux, l'or de ma chevelure, les parfums de mon vase, j'ai à mon tour célébré la Présence, un corps à toucher, adorer, un corps bien vivant. On s'est moqué de moi, on m'a presque chassée. Mon amour était indécent. ${ }^{22}$

En 1991, soit sept ans avant de remporter le prix Nobel de littérature, le romancier portugais José Saramago publie L'Evangile selon Jésus-Christ. Ce contre-évangile,

tout entière à la volupté. Mais c'est là une légende fausse et gratuite: et le Frère Albert, dans sa préface à l'évangile de saint Jean, nous affirme que la fiancée que le saint quitta pour suivre Jésus, resta vierge toute sa vie, et vécut, plus tard, dans la société de la Vierge Marie» (Jacques DE VORAGINE, La légende dorée, traduit du latin par Teodor DE WyZEWA, Paris, Seuil, 1998, p. 346).

(15) Marguerite Yourcenar, «Madeleine ou le salut», dans: Feux, Paris, Grasset, 1936, p. 114p. 115 .

(16) NiKos KaZANTZAKI, La dernière tentation du Christ, traduit du grec par Michel SAunier, Paris, Pocket, 1959 (1955), p. 339.

(17) Jacqueline Roux, Marie Reynès-Monlaur, une figure catholique du XXe siècle, Paris, Pierre Téqui, 2006.
(18) Marie Reynès-Monlaur, Le rayon, scènes évangéliques, Paris, Plon, 1911 (1902), p. 54.

(19) Bernard-G. Landry, Judas et Marie-Madeleine, correspondance intime, Pantin, Le Temps des Cerises, 2001, p. 56.

(20) BernaRD-G. LANDRY, op. cit., p. 34.

(21) Kelen a, notamment, commenté des textes dédiés à la sainte (Offrande à Marie-Madeleine, Paris, La Table Ronde, 2001) et rédigé la préface de la réédition de Lacordaire chez Million (HENRI LACORDAIRE, Sainte Marie-Madeleine, préfacé par Jacqueline KelEn, Grenoble, Jérôme Millon, 1998).

(22) JaCqueline Kelen, Marie-Madeleine, un amour infini, Paris, Albin Michel, 1992 (1982), p. 50 . 
largement controversé dans un Portugal encore très attaché à la foi catholique, décida de l'installation de son auteur à Lanzarote. Biographie fictive de Jésus, le roman retrace son existence, de sa conception au Golgotha. Jésus passe son adolescence dans le désert, avec, pour seul compagnon, l'incarnation humaine de Satan: Pasteur. Jeune adulte, il regagne la civilisation et blessé aux pieds, demande asile dans une maison de Magdala. Saramago nous livre alors sa lecture de l'onction. Une prostituée, Marie, ouvre sa porte à Jésus, le soigne de ses baumes et s'offre à lui. Les versets du Cantique des Cantiques scandent chaque geste des amants, qui passent de la parole d'amour au geste d'amour et ouvrent le rapport charnel à une dimension mystique. Cette scène marque la réconciliation de Jésus avec un corps que son mentor lui avait appris à mépriser. Une réconciliation qui permettra à Jésus d'entamer sa vie publique. Si l'écrivain ne mentionne pas les cheveux de la Magdaléenne durant l' «onction», les pages qui précèdent la rencontre du Christ et de Marie sont révélatrices du statut de la chevelure féminine, dans l'imaginaire du Portugais. Jésus se repose sur la rive du Jourdain et entend, sans l'apercevoir, une femme chanter. Le narrateur imagine alors la réaction de la baigneuse, si elle venait à découvrir le jeune homme...

Avec quelques paroles ou sans parole, la femme se dévêtira de nouveau, et quand sera arrivé ce qu'on doit toujours attendre de ce genre de rencontre, elle lui retirera ses sandales avec un soin extrême, elle guérira les plaies en déposant sur chaque pied un baiser et en les enveloppant ensuite, comme un œuf ou un cocon, dans sa propre chevelure humide. ${ }^{23}$

Mot pour mot, ce récit correspond à la rencontre du Messie et de Madeleine, le sexe de la prostituée succédant à la «chevelure humide» de la chanteuse ${ }^{24}$. Dans L'évangile selon Jésus-Christ, la chevelure de l'onction incarne une sexualité féminine valorisée.

Après le repas chez le Pharisien, plusieurs auteurs décrivent également le banquet de Béthanie. La scène affirme implicitement l'intellection de la disciple. Consciente à la fois de l'imminence de la Passion et de la véritable nature de Jésus, Madeleine anticipe son extrême-onction et le sacre roi, en lui versant de l'huile parfumée sur le front $^{25}$. Rien dans Jn 12, 1-11 ne permet donc d'assimiler la chevelure à l'expression du repentir. La popularité de la version lucanienne explique sans doute la régulière «contamination» de la deuxième onction.

En 1997, Pierrette Brès, écrivain française largement périphérique, publie Marie de Magdala ou la vie révélée de Marie-Madeleine. Littérairement très pauvre, ce roman constitue néanmoins une parfaite illustration de cette contamination. Alors que Madeleine a déjà effectué une première onction (aucune raison donc de combiner le message des deux épisodes, comme le font les partisans de l'onction unique), la jeune femme relâche ses cheveux, en signe «de deuil, de pénitence, de soumission» ${ }^{26}$, lorsqu'elle effectue l'onction des rois.

Indéniablement donc, malgré les quelques remises en question que nous avons tenu à souligner, la chevelure reste associée à l'expression du repentir dans la litté-

(23) José Saramago, L'Evangile selon Jésus-Christ, traduit du portugais par Geneviève LEIBRICH, Paris, Editions du Seuil, 1992 (1991), p. 287.

(24) Cette interprétation apparaît d'autant plus plausible si l'on sait que les pieds symbolisent les organes sexuels dans la civilisation judéo-chrétienne. «Nella cultura orientale et nella Bibbia [i piedi] contengono anche un richiamo sessuale, in quanto esprimono radicamento alla terra. Nell'Antico Testamento, a volte, i piedi servono come un eufe- mismo per designare gli organi sessuali» (LILIA SEBASTIANI, Il personaggio evangelico di Maria di Magdala e il mito della peccatrice redenta nella tradizione occidentale, Brescia, Queriniana, 1992, p. 60).

(25) Certains auteurs déplacent ce sacre royal à l'onction de Galilée.

(26) Pierrette Brès, Marie de Magdala ou la vie révélée de Marie-Madeleine, Paris, Michel Lafon, 1997 , p. 143-p. 144. Nous soulignons. 
rature contemporaine, et ce même en dehors du célèbre épisode. En 1913, Maurice Maeterlinck consacre un drame au destin de la sainte pécheresse. Lorsque l'héroïne de Marie-Magdeleine s'approche pour la première fois du Christ, son élégant chignon $s^{\prime}$ effondre ${ }^{27}$. Ses cheveux dénoués, dont la simplicitée ${ }^{28}$ s'oppose à l'artifice des riches coiffures passées, traduisent désormais sa contrition.

Dans Ma vie de Jésus (2005), le cinéaste et écrivain français Eduardo Manet envisage une attitude plus radicale. Son héroïne se coupe les cheveux le jour de sa conversion $^{29}$. Ce sacrifice symbolique d'une sexualité condamnée n'est pas totalement étranger à la tradition magdaléenne. Nous en avons trouvé trace dans l'illustration d'un Livre de la Passion du XIV ${ }^{\mathrm{e}}$ siècle ${ }^{30}$, dans un tableau de Caspar de Crayer $^{31}$, ainsi que dans un poème de Gabriel Vicaire ${ }^{32}$.

Corollaire évident de l'avilissement de la chevelure dans l'expression du repentir: les longues mèches restent intimement liée à la séduction de la Madeleine contemporaine. Bien avant le raz-de-marée médiatique suscité par le Da Vinci Code (2003) de Dan Brown, la romancière américaine Katherine Neville intègre les protagonistes des évangiles dans un thriller ésotérique, Le cercle magique (1998). Alors que dans la version de Neville, Madeleine ne se rend coupable que des péchés d'orgueil et de vanité $^{33}$, sa chevelure conserve de nets relents de luxure.

Sa chevelure abondante, dans l'éclat du soleil, composait un arc-en-ciel de nuances chatoyantes cascadant jusque sur ses épaules, avec une touche de provocation qui avait poussé les anciens, et même certains des disciples, à la considérer comme un ornement inutile et dangereux, politiquement incorrect, de l'entourage du maitre. ${ }^{34}$

Daniel Arasse ${ }^{35}$ souligne en effet le caractère ambigu de la «femme en cheveux», dont le négligé peut traduire, comme nous l'avons vu, le renoncement à la coquetterie, mais aussi la liberté de mœurs.

La métonymie sexuelle explique également la violence manifestée envers sa chevelure par les ennemis de la Magdaléenne. Dans La dernière tentation du Christ, Barrabas «empoigne Madeleine par ses tresses» dans la scène de la femme adultère ${ }^{36}$, tout comme Saül dans l'épisode onirique de la fin du roman, avant de l'assassiner ${ }^{37}$.

En 1946, Robert Graves publie un véritable hapax au sein de la littérature «christique» de ces dernières décennies. Alors que la tendance générale va vers l'assimilation du message évangélique aux cultes matriarcaux ${ }^{38}$, et, par conséquent, célèbre la fécondité et la sexualité, King Jesus dévalorise totalement la sensualité de la sainte. La

(27) Maurice Maeterlinck, Marie-Magdeleine, Paris, Eugène Fasquelle, 1913, p. 104.

(28) Diane Apostolos-Cappadona rappelle que les cheveux relâchés symbolisent l'énergie spirituelle, ce qui conviendrait parfaitement à ces moments-clés du parcours magdalénien où l'héroïne accède au religieux et au mystique («Images, Interpretations, and Traditions: a Study of Magdalene», dans Jane KopAs, Interpreting Tradition, the Art of Theological Reflexion, Chico, Scholar Press, 1983, p. 111).

(29) Edouardo Manet, Ma vie de Jésus, Paris, Grasset, 2005, p. 167.

(30) Dans un Livre de la Passion de la fin du XIV ${ }^{e}$ siècle, une illustration montre Marie-Madeleine se coupant les cheveux avec la légende «Marie Magdaleine coppe ses cheveux et offrit contrition» ( $\mathrm{S}$. HASKINS, op. cit., p. 150).
(31) Caspar de Crayer, Marie-Madeleine en vanité, première moitié du XVII ${ }^{\mathrm{e}}$ siècle, Valenciennes, Musée des Beaux-Arts.

(32) Gabriel Vicaire, Marie-Madeleine, Paris, Lemerre, 1889, p. 18.

(33) Katherine Neville, Le cercle magique, traduit de l'anglais par Gilles Morris-Dumoulin, Paris, Pocket, 2005 (1998), p. 254.

(34) Katherine Neville, op. cit., p. 50.

(35) Daniel Arasse, «La toison de Madeleine», dans On n'y voit rien, descriptions, Paris, Gallimard, 2003, p. 115-p. 116.

(36) Nikos Kazantzaki, op. cit., p. 177.

(37) NiKOS KaZANTZAKI, op. cit., p. 465.

(38) Katherine Rondou, Marie-Madeleine, Soi féminin complet dans le roman contemporain après 1950, dans «Cahiers d'Histoire des Littératures Romanes», 2006, V. 30, p. 461-p. 481. 
femme de Magdala n'incarne plus un type de sexualité généralement condamné - la prostitution - mais la sexualité féminine en général.

Humaniste célèbre dans les milieux anglo-saxons ${ }^{39}$, Robert Graves puise son inspiration dans sa connaissance érudite des civilisations antiques. Il détourne textes sacrés ou historiques, et éclaire de façon tout à fait personnelle la vie de Jésus et de ses compagnons. Il s'intéresse à la déesse antique de la Méditerranée à partir de 1944 (il lui consacre l'un de ses essais, White Goddess), et cherche à confronter le Christ au culte de la Grande Mère

Afin de permettre à l'homme d'échapper au cycle de la naissance et de la mort ${ }^{40}$, donc au pouvoir de la Femme, de la Grande Mère, le Christ doit soumettre sa représentante principale, Marie la Coiffeuse ${ }^{41}$. Il saisit la reine des prostituées par les cheveux pour l'exorciser.

Qu'ai-je à faire du Vieil Adam qui murmure dans la poussière? Un Nouvel Adam arrive au nom du Très-Haut pour accomplir, pour lier la Femme avec sa propre chevelure, pour charger l'Adversaire de Dieu de chaînes adamantines. Dans le Vieil Adam, tout meurt; dans le Nouveau, tout vivra. ${ }^{42}$

A l'opposé, plusieurs auteurs insisteront sur la charge positive des longs cheveux de la jeune femme, incarnation d'une féminité valorisée. Non seulement dans les pages consacrées à l'onction, comme nous l'avons vu, mais aussi lors de la Passion. Dans les oeuvres de Kelen et Yourcenar, la douceur de la chevelure magdaléenne offre un contraste frappant avec la violence des bourreaux.

Mais elle n'avait jamais eu, Marie, que ses mains, ses larmes et ses cheveux pour panser les rêves des hommes enténébrés. ${ }^{43}$

En vain, j'ai versé sur ses pieds l'onde oxygénée de ma chevelure [...]. ${ }^{44}$

Les épisodes déjà évoqués appartiennent aux évangiles et remontent au I ${ }^{\mathrm{er}}$ siècle. La vie «post-testamentaire» de la sainte, plus tardive, date de la fin du IX ${ }^{e}$ siècle. Placée sur un bateau sans gouvernail par ses opposants juifs, Madeleine accoste miraculeusement en Provence. Après la conversion de nombreux païens, la jeune femme se retire dans la solitude d'une grotte, la Sainte-Baume. Les littératures homilétique et hagiographique médiévales attribueront cette retraite à un brûlant désir de communier avec Dieu ${ }^{45}$. Plus tard, l'iconographie, peu attachée à la représentation de la Madeleine au désert avant la Renaissance ${ }^{46}$, popularisera l'image, devenue emblématique, de la pénitente expiant, dans l'isolement et les privations, une vie de débau$\mathrm{che}^{47}$. Certes, l'ermite et sa chevelure-cilice auront un impact non négligeable sur la littérature, et ce jusqu'au $\mathrm{Xx}^{\mathrm{e}}$ siècle. Toutefois, les auteurs contemporains renouent

(39) Malheureusement peu traduit, Graves souffre d'un manque de visibilité dans la sphère francophone.

(40) Robert Graves, King Jesus, traduit de l'anglais par Claude SEBAN, s.l., Stock, 1993 (1946), p. 304.

(41) Robert Graves, op. cit., p. 332-p. 333.

(42) Robert Graves, op. cit., p. 349.

(43) Jacqueline Kelen, op. cit., p. 129.

(44) Marguerite Yourcenar, op. cit., p. 120.

(45) Elisabeth Pinto-Mathieu, Marie-Madeleine dans la littérature du Moyen-Âge, Paris, Beau- chesne, 1997 , p. 38.

(46) LOUIS RÉAU, «Madeleine», dans Iconographie de l'art chrétien, Paris, Presses Universitaires de France, 1958, p. 846-p. 859.

(47) Pécheresse repentie, certes, mais qui ne perd rien de ses charmes passés. Françoise Bardon a souligné la persistance de la sensualité de l'ermite dans la peinture baroque (F. BARDON, Le thème de la Madeleine pénitente au XVII siècle en France, dans «Journal of the Warburg \& Courtauld Institutes», 1968, V. 31, p. 278-p. 306). 
parfois avec les homélies et Vitae médiévales. L'amante éplorée se substitue à l'ascète, l'attribut de la féminité et de l'amour à la robe de bure. Pierre-Marie Beaude, docteur en théologie à l'université de Metz, en donne un parfait exemple. Dans Marie la Passante (1999), la chevelure rasée traduit le deuil et non l'expiation.

Je ne pleure pas sur ma vie de putain, non, mais sur le seul amour qu'il m'a été donné de vivre. J'ai rasé mes cheveux. [...]. Je me moque bien d'être laide ou belle, mon prince saura voir la beauté à travers la laideur, comme Dieu sut trouver beau le fils lié comme un agneau sur l'autel du mont Moriyya. ${ }^{48}$

Le roman a fait l'objet d'une adaptation théâtrale, où nous retrouvons un souci identique de réhabilitation. Contrairement à la légende, Marie de Magdala ne renie pas sa féminité, ne voile pas sa chevelure.

On dit que je me suis retirée dans un lieu discret pour pleurer mes péchés, et que je porte un voile sur la tête... ${ }^{49}$

Reste une dernière composante de l'attribut magdalénien qui, à notre connaissance, n'a pas suffisamment retenu l'attention de la critique: la couleur des cheveux. Depuis le Moyen-Âge, l'iconographie offre à la chevelure de la sainte toutes les nuances, des plus sombres aux plus lumineuses ${ }^{50}$. Aucun historien de l'art ne semble cependant avoir cherché à décrypter ce choix, en tenant compte évidemment de l'évolution des canons esthétiques et des éventuelles obsessions personnelles de l'artiste. Certes, plusieurs études mentionnent une prédilection pour les Madeleines rousses, mais sans qu'aucun dépouillement de corpus n'étaie cette affirmation. Mary M. Rowan, par exemple, situe au XVI ${ }^{\mathrm{e}}$ siècle la multiplication des Madeleines ermites rousses. «Ce ne sont plus les cheveux dorés du Moyen-Âge, mais, d'après l'intervention du Titien, des cheveux roux. (Il n'est pas sans intérêt de se souvenir du fait que les sorcières de l'époque sont souvent décrites comme ayant des cheveux roux, couleur de flamme) $\gg^{51}$. Françoise Gaillard n'hésite pas à citer «la luxuriante chevelure d'un blond vénitien ${ }^{52}$ au rang des attributs magdaléniens. Xavier Fauche, dans un ouvrage de vulgarisation, affirme également que «Marie-Madeleine est, en général, représentée avec de longs cheveux roux $»^{53}$. Diane Apostolos-Cappadona évoque les «flowing red hair» ${ }^{54}$ parmi les traits permettant l'identification de la sainte, à travers ses différentes représentations iconographiques. A leur suite, nous devons reconnaî-

(48) Pierre-Marie Beaude, Marie la Passante, Paris, Desclée de Brouwer, 1999, p. 74.

(49) Pierre-Marie Beaude, Marie la Passante. Cette adaptation théâtrale n'a fait l'objet d'aucune publication: nous remercions Rachel Boulenger, qui incarna l'héroïne dans la mise en scène d'Eric Lorvoire, lors du festival d'Avignon de 2000, de nous en avoir transmis une copie.

(50) Songeons, par exemple, à la Madeleine blonde du Retable d'Issenbeim de Matthias Grünewald (1512-1515, Colmar, Unterlinden), à la Madeleine aux cheveux noirs de Georges de La Tour ( $\mathrm{La} M a$ deleine pénitente, vers 1638-1643, New York, Metropolitan Museum of Art), à la pénitente brune de Johan Moreelse (Marie-Madeleine pénitente, XVII siècle, Caen, Musée des Beaux-Arts), à la rousse du Christ dans la maison de Marthe et Marie d'Alessandro Allori (XVI ${ }^{e}$ siècle, Vienne, Kunsthistorisches
Museum) etc.

(51) MARY M. RowAN, «Problème et paradoxe: l'image de la Madeleine dans les écrits des grandes abbesses au XVII siècle», dans Yves GIRAUD, L'image de la Madeleine du XV au XIX siècle, Fribourg, Editions Universitaires Fribourg, 1996, p. 199. Nous supposons que l'auteur se réfère à la Madeleine pénitente (vers 1530).

(52) Françoise GaIllard, Marie-Madeleine et l'art contemporain, dans «Art Absolument, les cahiers de l'art d'hier et d'aujourd'hui», hiver 2005, V. 11, p. 38 .

(53) Xavier Fauche, Roux et rousses, un éclat très particulier, Paris, Gallimard, 1997, p. 40.

(54) Diane Apostolos-Cappadona, «Mary Magdalene, Saint», dans Encyclopedia of Women in Religious Art, New York, Continuum, 1996, p. 244. 
tre que la majorité des tableaux illustrant les ouvrages consacrés à la sainte ont pour modèles des rousses ${ }^{55}$. Choix inconscient - mais ô combien révélateur - de l'éditeur ou réelle dominante, cette question mérite, à notre avis, de retenir l'attention des spécialistes.

Mais confessons que le problème n'a pas trouvé plus d'écho auprès des historiens de la littérature que des historiens de l'art. Il semblerait qu'aucune étude du personnage littéraire de la Madeleine ne s'attarde sur l'éventuelle signification de sa couleur de cheveux.

En 2007, Valérie André a publié un essai sur la valeur symbolique du roux en Occident, sans doute l'ouvrage actuellement le plus complet sur cette problémati$q e^{56}$. Elle retrace l'émergence des préjugés associés aux roux - méchanceté, avarice, etc. - et analyse leur adoption en littérature. Parmi ces nombreux traits péjoratifs, la luxure convient certainement le mieux à la jolie pécheresse. Les travaux de Mireille Dottin-Orsini sur la culture fin-de-siècle rejoignent les conclusions de Valérie André, «à lire les romans de l'époque, on pourrait croire que les prostituées sont toutes rousses sans exception, grâce il est vrai à la potasse et au henné plus qu'à la nature» ${ }^{57}$.

Contrairement à ce que nous avons constaté pour le XIX ${ }^{e}$ siècle ${ }^{58}$, fidèle au cliché de la rousse luxurieuse, les $\mathrm{XX}^{\mathrm{e}}$ et $\mathrm{XXI}^{\mathrm{e}}$ siècles établissent un contraste entre le stéréotype et la véritable personnalité de l'héroïne. Ils invitent le lecteur à reconsidérer la chevelure, et la féminité en général. Le roman de Beaude, particulièrement frappant de ce point de vue, attribue presque tous les traits de la femme fatale décadente (la chevelure rousse $^{59}$, les yeux verts ${ }^{60}$, l'assimilation aux félins ${ }^{61}$ ) à Marie la Passante, pour aussitôt nier leur caractère destructeur. Il souligne la quête d'indépendance et la douceur de la jeune femme.

Ma chevelure rousse me faisait femme lionne. Mes yeux bleu-vert rameutaient les couleurs tendres et dures de la mer phénicienne. Il m'arrivait de m'endormir dans mes longs cheveux, si soyeux. Allongée sur la plage ou bien sur la terrasse d'une chambre haute, je les disposais autour de moi, ils me faisaient une conque douce, et je restais ainsi à regarder le jour se défaire, monter la lune et suivre indéfiniment son chemin discret dans le ciel. ${ }^{62}$

Dan Brown, dans un registre bien différent, cherche également à réhabiliter Madeleine et sa sexualité. Le romancier américain publie en 2003 «le» best-seller de la littérature populaire magdaléenne, le $\mathrm{Da}$ Vinci Code. Dans ce thriller ésotérique, la sainte est l'épouse de Jésus, la mère de son enfant, sa disciple par excellence. Une situation intolérable pour certains apôtres qui, au lendemain de la Passion, accusent

55 Luca Giordano, Marie-Madeleine comme vanité, vers 1690, Duinkerken, Musée des BeauxArts; Jan Gossaert Mabuse, Portrait de Dame en Marie-Madeleine, vers 1478, Anvers, Musée Mayer van den Bergh; Frederick Sandys, Marie-Madeleine, 1859, Delaware, Art museum; Jules-Joseph Lefebvre, Marie-Madeleine dans la grotte, 1876, SaintPétersbourg, Ermitage; Domenico Feti, Sainte Marie-Madeleine pénitente, 1615, Boston, Museum of Fine Arts, etc.

(56) VALÉRIE ANDRÉ, Réflexions sur la question rousse, bistoire littéraire d'un préjugé, Paris, Tallandier, 2007.

(57) Mireille Dottin-Orsini, Cette femme qu'ils disent fatale, textes et images de la misogynie fin-desiècle, Paris, Grasset, 1993, p. 324.

(58) Katherine Rondou, Le thème de sainte Marie-Madeleine dans la littérature d'expression fran- çaise, en France et en Belgique, de 1814 à nos jours, thèse de doctorat présentée sous la direction de Michel BastiaEnsen, Université Libre de Bruxelles, 2006.

(59) La femme fatale fin-de-siècle est souvent rousse (M. DotTin-Orsini, op. cit., p. 324-p. 326).

(60) Les yeux des personnages sadiques dans le bas romantisme sont habituellement verts (MARIO PRAZ, La chair, la mort et le diable dans la littérature du XIX siècle, le romantisme noir, traduit de l'italien par Constance Thompson Pasquali, Paris, Denoel, 1977, p. 270).

(61) Bram Dijkstra, Les idoles de la perversité, figures de la femme fatale dans la culture fin-de-siècle, traduit de l'américain par José Kamoun, Paris, Seuil, 1992, p. 300 et suivantes.

(62) Pierre-Marie Beaude, op. cit., p. 30. La scène est identique dans la pièce. 
la jeune femme de prostitution, afin de l'éloigner de la direction de l'Eglise naissante. Madeleine se réfugie en France et dirige, dans l'ombre, la véritable communauté chrétienne... comme le feront ses descendants, les héritiers du Christ. A ceux-ci, elle lègue l'enseignement de son époux... et ses cheveux roux, signe d'élection et non d'infamie.

Réhabilitée également la rousseur de la Madeleine de Landry, mais sans pour autant faire l'économie du cliché. Consciente que sa «rouquinitude» fut souvent interprétée comme un signe de malédiction ${ }^{63}$, Marie-Madeleine saura gré à Judas de la réconcilier avec cette partie d'elle-même.

Je garde au fond des yeux et du fond du cœur l'image flamboyante de tes cheveux dénoués qui jettent leur lumière d'incendie sur cette coulée de taches de rousseur descendant de ton visage pour se répandre sur toute ta peau blanche, comme une voie lactée entre les deux majestueux soleils de tes seins, qui déroulerait la carte de ton firmament et se perdrait au bas de ton ventre, parmi les perles de rosée de ton désir serties dans un entrelacs de fils d'or rouge. (Lettre de Judas à Madeleine) ${ }^{64}$

Je voudrais te prendre dans mes doigts pour que tu puisses me caresser comme tu le faisais, souviens-toi, quand ton doigt parcourait ma peau en allant d'une de mes maudites taches de rousseur à l'autre comme on suit sur le Livre, d'une lettre à l'autre, avec la petite main d'ivoire du Yad, et que tu me récitais «Le cantique des cantiques» comme si tu en lisais les strophes entre mes seins ou sur mes cuisses, et tout à coup j'étais guérie de la honte d'être une de ces méchantes rouquines qui ont pris le soleil à travers une passoire et je ressentais, au contraire, le fierté de ma peau blanche comme un vélin d'agneau où chacun peut lire toute la poésie du monde. (Lettre de Madeleine à Judas) ${ }^{65}$

Encore une fois, la valorisation de la chevelure, et de la chevelure rousse en particulier, va de pair avec une acceptation de la sensualité féminine. Ici, comme dans quelques autres romans contemporains ${ }^{66}$, la sexualité de la sainte se prolonge au-delà de la conversion. Madeleine devient la maîtresse de Judas, la compagne d'une nuit de Jésus, et la partenaire occasionnelle des apôtres, sans que cela ne remette en question, ni la crédibilité de sa conversion, ni son élévation spirituelle.

Si l'inventaire des Madeleines rousses se révèle qualitativement intéressant de par sa remise en question du stéréotype éculé de la rousse lubrique, il demeure quantitativement pauvre et compromet radicalement les affirmations évoquées plus haut. La littérature contemporaine, à la suite du XIX ${ }^{\mathrm{e}}$ siècle, témoigne au contraire d'une dominante de la chevelure blonde.

Demeure cependant un problème de perception que nous désirons rapidement signaler. Tous les individus n'opèrent pas la même distinction entre des cheveux cuivrés, auburn, blond vénitien (le «rosso veneziano» des Italiens). «A strawberry blond, overlooked as ordinary in predominatly light-haired populations, is perceived as a redhaired in predominatly dark-haired population ${ }^{\$ 7}$. En témoignent les hérö̈nes de Marie Reynes-Monlaur et de l'abbé Auguste Lefranc, auteur en 1912 du roman Marie de Magdala. Lors de l'onction, Reynes-Monlaur souligne la «beauté blonde de Madeleine», pour s'arrêter quelques lignes plus bas sur ses «longs cheveux de cet or roux si rare en Orient» ${ }^{68}$. Que penser également de «l'épaisse chevelure couleur

(63) BERNARD-G.LANDRY, op. cit., p. 50.

(64) BERNARD-G.LANDRY, op. cit., p. 39.

(65) BERnARD-G.LANDRY, op. cit., p. 78.

(66) Katherine Rondou, Marie-Madeleine, Soi féminin complet, cit.

(67) Ruth Mellinkoff, «Red Hair and Ruddy
Skin», dans Outcasts: Signs of Otherness in Nothern European Art of the Late Middle Ages, Los Angeles, University of California Press, 1993, p. 147.

(68) Marie Reynes-Monlaur, op. cit., p. 37. Nous soulignons. 
ambre» ${ }^{69}$ de la Madeleine de Lefranc, dont le romancier évoquait auparavant les «opulentes nattes blondes» ${ }^{70}$ ?

Avant de passer aux Madeleines blondes et brunes, il ne nous semble pas inutile de signaler la juxtaposition relativement répandue de la sainte et du rouge, indépendamment de sa couleur de cheveux. Staude ${ }^{71}$, dans sa description d'une peinture sur bois de l'école de Rimini ${ }^{72}$ du XIV $^{\mathrm{e}}$ siècle, évoque la robe rouge qui caractérise Madeleine, sans pour autant préciser cette particularité ${ }^{73}$. De nombreux peintres ${ }^{74}$ témoi- $^{-}$ gnent effectivement de cet intérêt pour les Madeleines vêtues de rouge, mais encore une fois, il n'existe, à notre connaissance, aucune étude approfondie des couleurs associées à la sainte. Tout au plus pouvons-nous renvoyer le lecteur à l'essai de Ruth Mellinkoff, Outcasts. Il démontre la transposition du statut ambigu de la jeune femme dans plusieurs Crucifixion de l'Europe du Nord, par sa position - de dos ou de profil - et la richesse de son vêtement aux couleurs éclatantes ${ }^{75}$. Dans un autre chapitre, l'historienne de l'art nous éclaire sur la valeur symbolique du rouge. «Red, like yellow, has been continuously associated with whoredom and adultery, from the Harlot of Babylon and the biblical Rahab to the woman in Hawthorne's Scarlet Letter ${ }^{76}$. Nous comprenons aisément l'association de l'ancienne pécheresse à la couleur de la prostitution, dont nous avons trouvé trace chez nos auteurs. L'héroïne de Jacqueline Kelen, par exemple, échange sa robe bleue contre un vêtement rouge, lorsqu'elle «entre en prostitution ${ }^{77}$.

Mais Mellinkoff rappelle également l'ambivalence du rouge, symbole à la fois du sang du meurtrier et du martyre, du feu des enfers et du foyer ${ }^{78}$. Les travaux de Cazenave, Chevalier et Gheerbrand ${ }^{79}$ confirment cette double lecture, simultanément péjorative et valorisante. Le rouge est à la fois la couleur de la violence, de l'interdit, de la prostitution, de l'Enfer, et de la vie, de l'amour chrétien, de la flamme du Saint Esprit, du sang de la rédemption ${ }^{80}$. Une équivoque que les auteurs contemporains, notamment Pierrette Brès, se plaisent à exploiter.

Pendant son existence de pécheresse, [Marie] a toujours pris plaisir, par provocation, à se vêtir de rouge, la couleur que portent les prostituées. A l'aube de ce qu'elle espère être une

(69) Auguste Lefranc, Marie de Magdala, Paris, Lethielleux, 1912, p. 37.

(70) Auguste Lefranc, op. cit., p. 14.

(71) W. STAUde, Le crâne-calice au pied de la Croix, dans «La revue des arts», septembre 1954, V. 4, fasc. 3, p. 140.

(72) Ecole de Rimini, Crucifixion, $\mathrm{XIV}^{\mathrm{e}}$ siècle, Zurich, Kunsthaus.

(73) Marilena Mosco sera plus précise: «La Maddalena in rosso dai lunghi capelli biondi sciolti ai piedi della croce, come nell'esemplare giottesco di Strasburgo, sarà un leitmotiv destinato ad avere fortuna soprattutto nei vari esemplari di Bernardo Daddi, dei seguaci di Giotto e dei maestri senesi» (Marilena Mosco, La Maddalena tra sacro e profano, da Giotto a De Chirico, Milan, Arnoldo Mondadori editore, 1986, p. 102).

(74) Jan Mostaert, Descente de Croix, vers 15101516, Bruxelles, Musées royaux des Beaux-Arts; Bernardo Daddi, Crucifixion, 1338, Edimbourg, National Gallery of Scotland; Titien, Noli me tangere, 1512, Londres, National Gallery; Daniel Seghers, Couronne de fleurs avec Marie-Madeleine, première moitié du XVII siècle, Gand, Musée des Beaux-Arts; Carlo Crivelli, Marie-Madeleine, après 1480, Amsterdam, Rijksmuseum; James Ensor, Ma- deleine, 1887, Gand, Musée des Beaux-Arts; Simone Martini, Le portement de croix, vers 1284-1344, Paris, Musée du Louvre; Léon Spilliaert, Piéta, 1912, Ostende, Musée des Beaux-Arts; Masaccio, Crucifixion, 1426, Naples, Capodimonte, etc.

(75) Ruth MellinKoff, «The Magdalene», dans op. cit., p. 220-p. 222.

(76) Ruth Mellinkoff, «Colors», dans op. cit., p. 44.

(77) JaCqueline Kelen, op. cit., p. 12.

(78) Ruth Mellinkoff, «Colors», dans op. cit., p. 35 .

(79) Michel Cazenave, «Rouge», dans op. cit., p. 592-p. 594; Jean Chevalier et Alain GHeERBRANT, «Rouge», dans op. cit., p. 831-p. 833.

(80) Staude signale rapidement le lien privilégié de Madeleine et du sang de la rédemption (W. Staude, op. cit., p. 139-p. 140), dont nous avons trouvé trace dans la littérature, notamment lorsqu'à la Passion, le sang de Jésus coule sur la chevelure de Madeleine, prostrée au pied de la Croix. Voir Katherine Rondou, Le rapport de Marie de Magdala au sang de la rédemption dans les représentations littéraires contemporaines de la Passion, dans «Intersezioni, rivista delle idee», sous presse. 
nouvelle existence, c'est encore le rouge, écarlate, qui lui semble indiqué pour recevoir cette rédemption qu'elle souhaite ardemment. Car selon la tradition que connaît bien Marie, si le rouge est l'image symbolique du sang, du feu, de la mort, c'est aussi celle de la renaissance, de la vie et de l'amour. ${ }^{81}$

Ces différents extraits, associés à l'idée reçue ${ }^{82}$ d'une Marie-Madeleine forcément rousse, nous amènent à nous interroger sur l'origine de cette association. Ne pourrait-on envisager un lien privilégié entre la sainte et la couleur rouge - parfaitement compréhensible vu l'ambiguïté de la couleur, particulièrement adaptée au destin de la pécheresse repentie -, qui «déteint» du vêtement à la chevelure?

Contrairement à ce qui est communément admis donc, la majorité des auteurs contemporains qui précisent la couleur de cheveux de leur héroïne opte pour le blond.

De manière générale, les blonds évoquent, par leur couleur solaire, la beauté et la spiritualité ${ }^{83}$, traits éventuellement renforcés par l'assimilation classique de la chevelure blonde à l'or ${ }^{84}$, métal parfait dont l'éclat rappelle la lumière.

Habituellement, les auteurs s'inscrivent dans cette tradition. Nombre de chevelures dorées célèbrent le Christ lors de l'onction, comme nous l'avons déjà constaté dans le roman de Jacqueline Kelen.

Seul L'évangile selon Jésus-Christ charge la chevelure blonde d'une valeur négative. Le roman s'ouvre sur la description d'une Crucifixion, dont le narrateur cherche à identifier les protagonistes. Il hésite un instant sur l'identité du personnage féminin incarnant Madeleine. Son choix se porte tout d'abord sur une jeune femme, aux longs cheveux épars et au décolleté aguicheur ${ }^{85}$, avant de se fixer sur un autre personnage, dont la blondeur peut rappeler la débauche passée.

Nous ne prétendons pas affirmer que Marie-Madeleine ait été effectivement blonde, nous nous rallions juste au courant d'opinion majoritaire ${ }^{86}$ qui persiste à voir dans les blondes, qu'elles soient l'œuvre de la nature ou de la teinture, les instruments les plus efficaces du péché et de la perdition. Ayant été comme on le sait une pécheresse endurcie, la plus perdue d'entre les perdues, Marie-Madeleine devait aussi être blonde pour ne pas démentir les convictions, acquises à bon ou à mauvais escient, de la moitié du genre humain. ${ }^{87}$

Mais le narrateur rejette aussitôt cet argument, pour se tourner vers le véritable indice permettant l'identification de la sainte. Madeleine est celle qui lève vers le Christ un «regard plein d'un amour authentique et impérieux» ${ }^{88}$. Notons que dans le roman, Marie de Magdala - dont la sexualité, nous l'avons vu, est largement valorisée - aura des cheveux noirs...

Foncée également la chevelure de Madeleine dans Une femme innombrable, un roman de 2002 du théologien orthodoxe français Jean-Yves Leloup.

Te regarder, mon amour, quand se dénoue ta chevelure, et se voilent tes seins,

(81) Pierrette Brès, op. cit., p. 69-p. 70.

(82) Idée reçue en tous cas pour la littérature des $\mathrm{XIX}^{\mathrm{e}}, \mathrm{XX}^{\mathrm{e}}$ et XXI $\mathrm{XI}^{\mathrm{e}}$ siècles.

(83) Alain Gheerbrant et Jean Chevalier, «Blond», dans op. cit., p. 213.

(84) Alain Gheerbrant et Jean Chevalier, «Or», dans op. cit., p. 322-p. 325.
(85) José Saramago, op. cit., p. 14.

(86) Nous n'avons cependant pas trouvé trace de cet a-priori dans les dictionnaires de symboles consultés.

(87) José Saramago, op. cit., p. 16.

(88) José Saramago, op. cit., p. 16. 
et ta main blanche

sur cet océan noir. ${ }^{89}$

L'auteur célèbre la beauté de la jeune femme, sans exploiter les significations spécifiquement attribuées à la couleur noire en Occident ${ }^{90}$ (l'autorité, le deuil, les ténèbres, etc.). Ses contemporains ne procèderont pas différemment: les mèches noires ne constituent pas un sème particulier dans l'élaboration du personnage.

Ainsi que nous l'avions constaté pour le XIX ${ }^{e}$ siècle, le manque d'uniformité dans le choix des auteurs permet difficilement de synthétiser les éléments relevés. À l'exception de José Saramago, les auteurs mettant en scène des Madeleines blondes ou brunes ne cherchent visiblement pas à tirer parti des connotations liées à ces couleurs.

Cette analyse se révèle cependant fructueuse. D'une part, elle réévalue - dans les limites de notre corpus, évidemment - l'association consacrée de la sainte et de la chevelure rousse. D'autre part, elle remet en question les a-priori négatifs systématiquement associés à cette couleur.

Les $\mathrm{XX}^{\mathrm{e}}$ et $\mathrm{XXI}^{\mathrm{e}}$ siècles restent, comme les époques passées, sous le charme des longues mèches de la sainte, aujourd'hui encore l'une des principales reliques vénérées à Saint-Maximin ${ }^{91}$. Le plus souvent emblème de sa sensualité et de sa rédemption, la chevelure épouse toujours l'évolution spirituelle de Madeleine. Toutefois, celle-ci revêt une signification différente chez quelques auteurs contemporains et entraîne une relecture de l'attribut qui, sans être généralisée, marque un tournant non négligeable dans l'histoire du personnage littéraire. En effet, si l'existence de Madeleine se voit bouleversée par sa rencontre avec Jésus, son adhésion à la «Bonne Nouvelle» ne se concrétise pas nécessairement par un rejet de la sexualité, que celle-ci se prolonge ou non au-delà de la «conversion». Une acceptation de la sexualité féminine que traduit la chevelure de la sainte, non plus diabolique instrument de séduction ou matérialisation de son repentir, mais agent de son amour absolu.

KATHERINE RONDOU

(89) JeAn-Yves Leloup, Une femme innombrable, le roman de Marie-Madeleine, Paris, Albin Michel, 2002, p. 122.

(90) Alain Gheerbrant et Jean Chevalier, «Noir», dans op. cit., p. 272-p. 277.

(91) De longues mèches - mi-blond, mi-châtain pour Elisabeth Pinto-Mathieu (Marie-Madeleine dans la littérature du Moyen-Age cit., p. XII), blondes pour le Marquis de Virieu (Marquis de VIRIEU, Légendes et traditions provençales, Lyon-Paris, Albert-Vic, 1883, p. 4): encore un problème de perception... - contenues dans un vase de cristal. 